\title{
Generalized three-sided assignment markets: core consistency and competitive prices
}

\author{
Ata Atay • Francesc Llerena • Marina Núñez
}

the date of receipt and acceptance should be inserted later

\begin{abstract}
A generalization of the classical three-sided assignment market is considered, where value is generated by pairs or triplets of agents belonging to different sectors, as well as by individuals. For these markets we represent the situation that arises when some agents leave the market with some payoff by means of a generalization of Owen (1992) derived market. Consistency with respect to the derived market, together with singleness best and individual anti-monotonicity, axiomatically characterize the core for these generalized three-sided assignment markets. When one sector is formed by buyers and the other by two different type of sellers, we show that the core coincides with the set of competitive equilibrium payoff vectors.
\end{abstract}

Keywords Multi-sided assignment market · Consistency · Core · Competitive prices

Mathematics Subject Classification (2000) 91A12 - 91A40

\section{Introduction}

We consider a market with three-sectors where value is obtained by means of coalitions formed by agents of different sectors, that is, either triplets, pairs or individuals. Once the valuations of all these basic coalitions are known, a coalitional game is defined, the worth of an arbitrary coalition being the

A. $\operatorname{Atay}(\bowtie) \cdot$ M. Núñez

Dep. de Matemàtica Econòmica, Financera i Actuarial, Universitat de Barcelona, Av. Diagonal, 690, 08034 Barcelona, Spain

E-mail: aatay@ub.edu E-mail: mnunez@ub.edu

F. Llerena

Dep. de Gestió d'Empreses, Universitat Rovira i Virgili-CREIP, Av. de la Universitat 1, 43204 Reus, Spain

E-mail: francesc.llerena@urv.cat 
maximum worth that can be obtained by a partition of this coalition into basic ones.

Think, for instance, of one sector formed by firms providing landline telephone and internet service, on the second sector firms providing cable TV and on the third sector firms providing mobile telephone service. A triplet formed by one firm of each sector can achieve a profit by pooling their customers and offering them more services, but also a firm alone or a pair of firms of different sectors can attain some value.

These markets have already been considered in Tejada (2013) to see that agents of different sectors do not need to be complements and agents of the same sector do not need to be substitutes. Clearly, this class of coalitional games includes the classical three-sided assignment games of Quint (1991b) where value is only generated by triplets of agents belonging to different sectors. Another possible generalization of three-sided assignment games would be just assigning a reservation value to each individual and assuming that whenever an agent does not form part of any triplet then this agent can attain his/her reservation value, in the way Owen (1992) generalizes the classical two-sided assignment game of Shapley and Shubik (1972).

The difference between the generalized three-sided markets that we consider and the three-sided assignment markets with individual reservation values is that when an agent does not form part of a triplet in the optimal partition (that we will name optimal matching), apart from being alone in an individual coalition, he/she may form part of a two-player coalition with some agent belonging to a different sector and, in that case, the value of this twoplayer coalition may be larger than the addition of the individual reservation value of the two agents. As a consequence, ours is a wider class since it includes games that are not strategically equivalent to a Quint (1991b) three-sided assignment game. Nevertheless, as in the classical three-sided assignment games, these games may not be balanced (the core may be empty).

However, we present a subclass of balanced generalized three-sided assignment markets. Besides non-negativeness, two additional properties define this subclass: a) the worth of a triplet is the addition of the worths of the three pairs that can be formed with its members and b) there is an optimal partition such that, when restricted to each pair of sectors, is also optimal for the related two-sided market. This subclass of generalized three-sided assignment markets is inspired by the balanced subclass introduced by Quint (1991b) and the supplier-firm-buyer market of Stuart (1997), where also the value of a triplet is obtained by the addition of the value of some of the pairs that can be formed with its elements. However, in their classes, such a pair cannot attain its value if not matched with an agent of the remaining sector.

We restrict to the three-sided case to keep notation simpler, but all the arguments and results on the present paper can be extended to the multisided case.

In this class of generalized three-sided assignment markets, we introduce a reduced market at a given coalition and payoff vector, which represents the situation in which members outside the coalition leave the game with a 
predetermined payoff and the agents that remain in the market reevaluate their coalitional worth taking into account the possibility of cooperation with the agents outside. In the case of only two sectors, this reduced market coincides with the derived market defined by Owen (1992) for two-sided assignment markets with agents' reservation values.

Making use of consistency with respect to the derived market and two additional axioms, singleness best and individual anti-monotonicity, we provide an axiomatic characterization of the core on the domain of generalized three-sided assignment markets. Sasaki (1995) and Toda (2005) characterize the core on the domain of two-sided assignment markets by means of some monotonicity property that is not satisfied by the core in the three-sided case. The reason is that when we raise the value of a triplet, a pair or an individual in a three-sided market, the new market may fail to have core elements. This is why the previous characterizations cannot be straightforwardly extended to the three-sided case.

In the last part of the paper we consider that one of the sectors is formed by buyers and the others by sellers of two different types of goods. Each buyer can buy at most one good of each type and valuates all basic coalitions she/he can take part in. From these valuations we introduce the demand of a buyer, given a price for each object on sale. Then, as usual, prices are competitive if there exists a matching such that each buyer takes part in a basic coalition in its demand set, and prices of unsold objects are zero. We show that the set of payoff vectors related to competitive equilibria coincide with the core. This generalizes the result in Gale (1960) for two-sided assignment markets and Tejada (2010) for the classical multi-sided assignment markets where buyers are forced to acquire exactly one item of each type.

The paper is organized as follows. The model is described in Section 2. The derived consistency of the core and the nucleolus is proved in Section 3 , and an axiomatic characterization of the core is presented in Section 4 . Section 5 focuses on the case with one sector of buyers and two sectors of sellers of different type of goods to prove the coincidence of core elements and competitive equilibria payoff vectors. The Appendix contains some technical proofs.

\section{The model}

In this section, we introduce a generalized three-sided assignment market and its corresponding assignment game.

Let $\mathcal{U}_{1}, \mathcal{U}_{2}$ and $\mathcal{U}_{3}$ be three pairwise disjoint countable sets. A generalized three-sided assignment market consists of three different sectors, $M_{1} \subseteq \mathcal{U}_{1}$, $M_{2} \subseteq \mathcal{U}_{2}$, and $M_{3} \subseteq \mathcal{U}_{3}$ with a finite number of agents each, such that $N=$ $M_{1} \cup M_{2} \cup M_{3} \neq \emptyset$, and a valuation fuction $v$. The basic coalitions in this market are the ones formed by exactly one agent of each sector and all their 
possible subcoalitions. Let us denote by $\mathcal{B}$ this set of basic coalitions,

$$
\begin{aligned}
\mathcal{B} & =\left\{\{i, j, k\} \mid i \in M_{1}, j \in M_{2}, k \in M_{3}\right\} \\
& \cup\left\{\{i, j\} \mid i \in M_{r}, j \in M_{s}, r, s \in\{1,2,3\}, r \neq s\right\} \cup\left\{\{i\} \mid i \in M_{1} \cup M_{2} \cup M_{3}\right\} .
\end{aligned}
$$

The valuation function $v$, from the set $\mathcal{B}$ to the real numbers, $\mathbb{R}$, associates to each basic coalition its value $v(S)$.

Given a generalized three-sided assignment market $\gamma=\left(M_{1}, M_{2}, M_{3} ; v\right)$, for each non-empty coalition $S \subseteq N=M_{1} \cup M_{2} \cup M_{3}$ we can define a submarket $\gamma_{\mid S}=\left(M_{1} \cap S, M_{2} \cap S, M_{3} \cap S ; v_{\mid S}\right)$ where $\left(v_{\mid S}\right)(E)=v(E)$ for all $E \in \mathcal{B}^{S}=\{R \in \mathcal{B} \mid R \subseteq S\}$. Notice that if one of the sectors is empty, then this generalized three-sided assignment market is a two-sided assignment market with reservation values as introduced in Owen (1992).

Let $\emptyset \neq S \subseteq N$ be a coalition such that $S=S_{1} \cup S_{2} \cup S_{3}$ with $S_{1} \subseteq M_{1}$, $S_{2} \subseteq M_{2}$, and $S_{3} \subseteq M_{3}$. Then, a matching $\mu$ for $S$ is a partition of $S$ in coalitions of $\mathcal{B}^{S}$. Let $\mathcal{M}\left(S_{1}, S_{2}, S_{3}\right)$ be the set of all possible matchings for coalition $S=S_{1} \cup S_{2} \cup S_{3}$. A matching $\mu \in \mathcal{M}\left(S_{1}, S_{2}, S_{3}\right)$ is optimal for the submarket $\gamma_{\mid S}$ if $\sum_{E \in \mu} v(E) \geq \sum_{E \in \mu^{\prime}} v(E)$ for any $\mu^{\prime} \in \mathcal{M}\left(S_{1}, S_{2}, S_{3}\right)$. We denote by $\mathcal{M}_{\gamma}\left(S_{1}, S_{2}, S_{3}\right)$ the set of optimal matchings for the market $\gamma_{\mid S}$.

Given a generalized three-sided assignment market $\gamma=\left(M_{1}, M_{2}, M_{3} ; v\right)$, its corresponding generalized three-sided assignment game ${ }^{1}$ is a pair $\left(N, w_{\gamma}\right)$ where $N=M_{1} \cup M_{2} \cup M_{3}$ is the player set and the characteristic function $w_{\gamma}$ satisfies $w_{\gamma}(\emptyset)=0$ and for all $S \subseteq N$,

$$
w_{\gamma}(S)=\max _{\mu \in \mathcal{M}\left(S_{1}, S_{2}, S_{3}\right)}\left\{\sum_{E \in \mu} v(E)\right\},
$$

where $S_{1}=S \cap M_{1}, S_{2}=S \cap M_{2}$ and $S_{3}=S \cap M_{3}$. Notice that the game $\left(N, w_{\gamma}\right)$ is superadditive because it is a special type of partitioning game as introduced by Kaneko and Wooders (1982).

From now on, we denote by $\Gamma_{3-G A M}$ indistinctly the set of generalized three-sided assignment markets or games.

An outcome for a generalized three-sided assignment market will be a matching and a distribution of the profits of this matching among the agents that take part.

Given $\gamma=\left(M_{1}, M_{2}, M_{3} ; v\right)$, a payoff vector is $x \in \mathbb{R}^{N}$, where $x_{i}$ stands for the payoff of player $i \in N$. We write $x_{\mid S}$ to denote the projection of a payoff vector $x$ to agents in coalition $S \subseteq N$. Moreover, $x(S)=\sum_{i \in S} x_{i}$ with $x(\emptyset)=0$. A payoff vector $x \in \mathbb{R}^{N}$ is individually rational for $\gamma$ if $x_{i} \geq w_{\gamma}(\{i\})$ for all $i \in N$, and efficient if $x(N)=w_{\gamma}(N)$.

The core of a generalized three-sided assignment market $\gamma=\left(M_{1}, M_{2}, M_{3} ; v\right)$ is the core of the associated assignment game $\left(N, w_{\gamma}\right)$, where $N=M_{1} \cup M_{2} \cup$

\footnotetext{
${ }^{1}$ A game is a pair formed by a finite set of players $N$ and a characteristic function $r$ that assigns a real number $r(S)$ to each coalition $S \subseteq N$, with $r(\emptyset)=0$. The core of a coalitional game $(N, r)$ is $C(r)=\left\{x \in \mathbb{R}^{N} \mid \sum_{i \in N} x_{i}=r(N), \sum_{i \in S} x_{i} \geq r(S)\right.$ for all $\left.S \subseteq N\right\}$. A game is balanced if it has a non-empty core.

A game is said to be superadditive if for any two disjoint coalitions $S, T \subseteq N, S \cap T=\emptyset$, it holds $r(S \cup T) \geq r(S)+r(T)$.
} 
$M_{3}$. Then, a market $\gamma$ is balanced if its associated game $\left(N, w_{\gamma}\right)$ has a nonempty core. It is straightforward to see that this core is formed by those efficient payoff vectors that satisfy coalitional rationality for all coalitions in $\mathcal{B}$. Given any optimal matching $\mu \in \mathcal{M}_{\gamma}\left(M_{1}, M_{2}, M_{3}\right)$,

$$
C(\gamma)=\left\{x \in \mathbb{R}^{N} \mid x(N)=\sum_{E \in \mu} v(E) \text { and } x(E) \geq v(E) \text { for all } E \in \mathcal{B}\right\} .
$$

As a consequence, given any optimal matching $\mu$, if $x \in C(\gamma)$, then $x(E)=$ $v(E)$ for all $E \in \mu$. Since this class is a generalization of the classical threesided assignment games, the core may be empty.

The following two examples show that the class of generalized three-sided assignment games is indeed different from the class of classical three-sided assignment games. If we give values to some two-player coalitions in a classical three-sided assignment game with empty core (non-empty core), the core of the new generalized three-sided assignment game may become non-empty (empty). Moreover, we show that a generalized three-sided assignment game may not be strategically equivalent to any classical three-sided assignment game.

Example 1 Consider $M_{1}=\{1,2\}, M_{2}=\left\{1^{\prime}, 2^{\prime}\right\}$ and $M_{3}=\left\{1^{\prime \prime}, 2^{\prime \prime}\right\}$ and the three-sided assignment game taken from Quint (1991b) where the value of triplets is given by the following three-dimensional matrix $A$,

$$
\begin{aligned}
& 1^{\prime} 2^{\prime} \quad 1^{\prime} 2^{\prime} \\
& \begin{array}{c}
1 \\
1^{\prime \prime}
\end{array}\left(\begin{array}{cc}
0 & 0 \\
0 & 1
\end{array}\right) \quad \frac{1}{2^{\prime \prime}}\left(\begin{array}{ll}
0 & 1 \\
1 & 1
\end{array}\right),
\end{aligned}
$$

and the core is shown to be empty.

Define now a generalized three-sided market $\gamma_{1}=\left(M_{1}, M_{2}, M_{3} ; v_{1}\right)$ where $v_{1}(\{i, j, k\})=a_{i j k}$ for $(i, j, k) \in M_{1} \times M_{2} \times M_{3}, v_{1}\left(\left\{1,1^{\prime}\right\}\right)=1$ and $v_{1}(S)=0$ for any other $S \in \mathcal{B}$. Notice that $w_{\gamma_{1}}\left(\left\{1,1^{\prime}, 1^{\prime \prime}\right\}\right)=w_{\gamma_{1}}\left(\left\{1,1^{\prime}, 2^{\prime \prime}\right\}\right)=1$ and $x=(0,0 ; 1,1 ; 0,0) \in C\left(\gamma_{1}\right)$.

Moreover, the game $\left(N, w_{\gamma_{1}}\right)$, where $N=M_{1} \cup M_{2} \cup M_{3}$, is not strategically equivalent to any classical three-sided assignment game. Indeed, if there existed $d \in \mathbb{R}^{N}$ and a three-dimensional matrix $B$ such that $w_{\gamma_{1}}(S)=w_{B}(S)+\sum_{i \in S} d_{i}$ for all $S \subseteq N$, then

$$
1=w_{\gamma_{1}}\left(\left\{1,1^{\prime}\right\}\right)=w_{B}\left(\left\{1,1^{\prime}\right\}\right)+d_{1}+d_{2}=d_{1}+d_{2}
$$

which means either $d_{1}>0$ or $d_{2}>0$. If we assume without loss of generality that $d_{1}>0$, then we get a contradiction since $0=w_{\gamma_{1}}(\{1\})=w_{B}(\{1\})+d_{1}>$ 0 .

Example 2 Consider now a classical three-sided assignment game with a nonempty core given in Quint (1991b). It is defined by $M_{1}=\{1,2\}, M_{2}=\left\{1^{\prime}, 2^{\prime}\right\}$, 
$M_{3}=\left\{1^{\prime \prime}, 2^{\prime \prime}\right\}$ and the three-dimensional matrix $C$, where an optimal matching is in boldface:

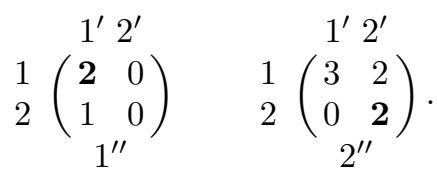

Define now a generalized three-sided market $\gamma_{2}=\left(M_{1}, M_{2}, M_{3} ; v_{2}\right)$ where $v_{2}(\{i, j, k\})=c_{i j k}$ for $(i, j, k) \in M_{1} \times M_{2} \times M_{3}, v_{2}\left(\left\{1,2^{\prime}\right\}\right)=v_{2}\left(\left\{2,1^{\prime}\right\}\right)=2$ and $v_{2}(S)=0$ for any other $S \in \mathcal{B}$. This implies that $w_{\gamma_{2}}\left(\left\{1,1^{\prime}, 2,2^{\prime}\right\}\right)=4$. If $x \in C\left(\gamma_{2}\right)$, we have $x_{1}+x_{1^{\prime}}+x_{2}+x_{2^{\prime}} \geq 4$ and hence $x_{1^{\prime \prime}}=x_{2^{\prime \prime}}=0$. Moreover, from $w_{\gamma_{2}}\left(\left\{1,1^{\prime}, 1^{\prime \prime}\right\}\right)=2$ we have $x_{1}+x_{1^{\prime}}=x_{1}+x_{1^{\prime}}+x_{1^{\prime \prime}}=2$. On the other side, from $w_{\gamma_{2}}\left(\left\{1,1^{\prime}, 2^{\prime \prime}\right\}\right)=3, x_{1}+x_{1^{\prime}}=x_{1}+x_{1^{\prime}}+x_{2^{\prime \prime}} \geq 3$, which leads to a contradiction and implies that $C\left(\gamma_{2}\right)=\emptyset$.

To conclude the discussion of the model, we introduce a subclass of generalized three-sided assignment markets. For the markets in this subclass, core allocations always exist.

\subsection{A subclass of markets with non-empty core}

We say a generalized three-sided assignment market is 2-additive if the three following conditions hold. The first one requires non-negativeness of the valuation function, with null value for single-player coalitions. Secondly, the valuation of each triplet $(i, j, k) \in M_{1} \times M_{2} \times M_{3}$ is the sum of the valuations of all pairs of agents in the triplet. Finally, we require the existence of an optimal matching that induces an optimal matching in each two-sided market. The reader will notice that the spirit of this class of 2-additive generalized threesided assignment markets, that we denote by $\Gamma_{3-G A M}^{a d d}$, is similar to that of the balanced classes of multi-sided assignment games in Quint (1991b) and Stuart (1997). In both cases, the authors impose that the worth of a triplet is the addition of some numbers attached to its pairs. The difference is that in their models a pair cannot attain its worth if not matched with a third agent of the remaining sector, while in our case there is an underlying two-sided market for each pair of sectors.

As in Quint (1991b), we will assume from now on that the market is square, that is $\left|M_{1}\right|=\left|M_{2}\right|=\left|M_{3}\right|$. Let us introduce some notation: given a generalized three-sided assignment market $\gamma=\left(M_{1}, M_{2}, M_{3} ; v\right)$, for all $r, s \in\{1,2,3\}$, $r<s$, we consider the two-sided market $\gamma^{r s}=\left(M_{r}, M_{s} ; v_{\mid \mathcal{B}^{M_{r}} \cup M_{s}}\right)$. Then, we denote by $\mathcal{M}_{\gamma^{r s}}\left(M_{r}, M_{s}\right)$ the set of optimal matchings for the two-sided market $\gamma^{r s}$, that is, partitions of $M_{r} \cup M_{s}$ in mixed pairs and singletons that maximize the sum of the valuations of the coalitions in the partition. And $C\left(\gamma^{r s}\right)$ stands for the core of the underlying two-sided assignment game $\left(M_{r} \cup M_{s}, w_{\gamma^{r s}}\right)$.

Given a matching $\mu \in \mathcal{M}\left(M_{1}, M_{2}, M_{3}\right)$ and two different sectors $r, s \in$ $\{1,2,3\}, r<s$, the matching $\mu$ induces a matching $\mu^{r s}$ in the two-sided 
market $\gamma^{r s}$ simply by defining $E \in \mu^{r s}$ if there exists a basic coalition $E^{\prime} \in \mu$ such that $E=E^{\prime} \cap\left(M_{r} \cup M_{s}\right)$ and $E \neq \emptyset$.

Definition 1 A generalized three-sided assignment market $\gamma=\left(M_{1}, M_{2}, M_{3} ; v\right)$, with $\left|M_{1}\right|=\left|M_{2}\right|=\left|M_{3}\right|$, belongs to the class $\Gamma_{3-G A M}^{a d d}$ if and only if

1. $v \geq 0$ and $v(\{k\})=0$ for all $k \in M_{1} \cup M_{2} \cup M_{3}$,

2. $v(\{i, j, k\})=v(\{i, j\})+v(\{i, k\})+v(\{j, k\})$ for all $(i, j, k) \in M_{1} \times M_{2} \times M_{3}$,

3. there exists $\mu \in \mathcal{M}_{\gamma}\left(M_{1}, M_{2}, M_{3}\right)$ such that $\mu^{r s} \in \mathcal{M}_{\gamma^{r s}}\left(M_{r}, M_{s}\right)$ for all $r, s \in\{1,2,3\}, r<s$.

Conditions (1) and (2) imply that the valuation function $v$ is superadditive. Condition (3) requires that there is an optimal matching $\mu \in \mathcal{M}_{\gamma}\left(M_{1}, M_{2}, M_{3}\right)$ that induces an optimal matching in each bilateral market $\gamma^{r s}$, for $r<s$. Next proposition shows that the three conditions together guarantee that the core of any generalized three-sided assignment market in the class $\Gamma_{3-G A M}^{a d d}$ is nonempty.

Proposition 1 Each 2-additive generalized three-sided assignment market is balanced.

Proof Let $\gamma=\left(M_{1}, M_{2}, M_{3} ; v\right) \in \Gamma_{3-G A M}^{a d d}$ and let $\mu=\left\{E_{1}, E_{2}, \ldots, E_{p}\right\}$ be an optimal matching, $\mu \in \mathcal{M}_{\gamma}\left(M_{1}, M_{2}, M_{3}\right)$, such that $\mu^{r s} \in \mathcal{M}_{\gamma^{r s}}\left(M_{r}, M_{s}\right)$ for all $r, s \in\{1,2,3\}, r<s$. For all $r, s \in\{1,2,3\}, r<s$, and $l \in\{1,2, \ldots, p\}$, define $E_{l}^{r s}=E_{l} \cap\left(M_{r} \cup M_{s}\right)$ and notice that by definition $\mu^{r s}=\left\{E_{l}^{r s} \mid 0 \leq\right.$ $\left.l \leq p, E_{l}^{r s} \neq \emptyset\right\}$.

From Shapley and Shubik (1972), it is known that each two-sided assignment market is balanced. So, take core allocations $\left(x^{1}, y^{1}\right) \in C\left(\gamma^{12}\right),\left(x^{2}, z^{2}\right) \in$ $C\left(\gamma^{13}\right)$ and $\left(y^{3}, z^{3}\right) \in C\left(\gamma^{23}\right)$. We will see that $\left(x^{1}+x^{2}, y^{1}+y^{3}, z^{2}+z^{3}\right) \in C(\gamma)$.

By optimality of $\mu^{12}$, we have that if for some $l \in\{1,2, \ldots, p\}, E_{l}^{12}=\{i, j\}$, then $x_{i}^{1}+y_{j}^{1}=v(\{i, j\})$. Similarly, if $E_{l}^{12}=\{i\}$, for $i \in M_{1}$, then $x_{i}^{1}=0$; and if $E_{l}^{12}=\{j\}$ for some $j \in M_{2}$, then $y_{j}^{1}=0$. Analogous equalities are obtained for $E_{l}^{13}$ and $E_{l}^{23}$, for $l \in\{1,2, \ldots, p\}$.

Hence,

$$
\begin{aligned}
& \sum_{i \in M_{1}}\left(x_{i}^{1}+x_{i}^{2}\right)+\sum_{j \in M_{2}}\left(y_{j}^{1}+y_{j}^{3}\right)+\sum_{k \in M_{3}}\left(z_{k}^{2}+z_{k}^{3}\right)= \\
& \sum_{l=1}^{p}\left[\sum_{\{i, j\} \in E_{l}^{12}}\left(x_{i}^{1}+y_{j}^{1}\right)+\sum_{\{i, k\} \in E_{l}^{13}}\left(x_{i}^{2}+z_{k}^{2}\right)+\sum_{\{j, k\} \in E_{l}^{23}}\left(y_{j}^{3}+z_{k}^{3}\right)\right]= \\
& \sum_{l=1}^{p}\left[\sum_{\{i, j\} \in E_{l}^{12}} v(\{i, j\})+\sum_{\{i, k\} \in E_{l}^{13}} v(\{i, k\})+\sum_{\{j, k\} \in E_{l}^{23}} v(\{j, k\})\right]= \\
& \sum_{l=1}^{p} v\left(E_{l}\right)=w_{\gamma}(N) .
\end{aligned}
$$

Once proved efficiency, it only remains to prove coalitional rationality of the payoff vector $\left(x^{1}+x^{2}, y^{1}+y^{3}, z^{2}+z^{3}\right)$. Indeed, take any $\{i, j, k\} \in \mathcal{B}$ and notice that

$$
\begin{aligned}
x_{i}^{1}+x_{i}^{2}+y_{j}^{1}+y_{j}^{3}+z_{k}^{2}+z_{k}^{3} & =\left(x_{i}^{1}+y_{j}^{1}\right)+\left(x_{i}^{2}+z_{k}^{2}\right)+\left(y_{j}^{3}+z_{k}^{3}\right) \\
& \geq v(\{i, j\})+v(\{i, k\})+v(\{j, k\})=v(\{i, j, k\})
\end{aligned}
$$


where the inequality follows from the core constraints of $\left(x^{1}, y^{1}\right),\left(x^{2}, z^{2}\right)$ and $\left(y^{3}, z^{3}\right)$ in each two-sided market.

Similarly, if $\{i, j\} \in \mathcal{B}$, we may assume without loss of generality that $i \in M_{1}$ and $j \in M_{2}$, and hence, taking into account $x_{i}^{2} \geq v(\{i\})=0$ and $y_{j}^{3} \geq v(\{j\})=0$, we get

$$
x_{i}^{1}+x_{i}^{2}+y_{j}^{1}+y_{j}^{3}=\left(x_{i}^{1}+y_{j}^{1}\right)+x_{i}^{2}+y_{j}^{3} \geq v(\{i, j\}) .
$$

Finally, if $\{i\} \in \mathcal{B}$, let us assume without loss of generality that $i \in M_{1}$. Then $x_{i}^{1}+x_{i}^{2} \geq 0=v(\{i\})$ follows also from the individual rationality of $\left(x^{1}, y^{1}\right)$ and $\left(x^{2}, z^{2}\right)$.

In the above proposition we have deduced the existence of core elements for $\gamma \in \Gamma_{3-G A M}^{a d d}$ by operating with three core elements of the related two-sided markets. However, as the next example shows, there are 2-additive generalized three-sided markets where not all core elements can be obtained in this way.

Example 3 Let us consider a generalized three-sided asignment market $\gamma$ where $M_{1}=\{1,2\}, M_{2}=\left\{1^{\prime}, 2^{\prime}\right\}$ and $M_{3}=\left\{1^{\prime \prime}, 2^{\prime \prime}\right\}$. The value of individual coalitions is null, the value of those basic coalitions formed by a pair of agents is given by

$$
\begin{aligned}
& 1^{\prime} 2^{\prime} \quad 1^{\prime \prime} 2^{\prime \prime} \quad 1^{\prime \prime} 2^{\prime \prime} \\
& \begin{array}{l}
1 \\
2
\end{array}\left(\begin{array}{cc}
\text { (4) } & 6 \\
0 & (4)
\end{array}\right) \quad \begin{array}{l}
1 \\
2
\end{array}\left(\begin{array}{cc}
6 & 9 \\
1 & 5
\end{array}\right) \quad \begin{array}{l}
1^{\prime} \\
2^{\prime}
\end{array}\left(\begin{array}{cc}
(2) & 0 \\
8 & 7
\end{array}\right) \text {, }
\end{aligned}
$$

and the value of triplets is given by the following three-dimensional matrix

$$
\begin{aligned}
& 1^{\prime} 2^{\prime} \quad 1^{\prime} 2^{\prime}
\end{aligned}
$$

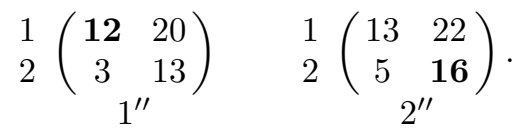

The reader can check that the above values define a 2 -additive generalized three-sided market. Optimal matchings of the underlying two-sided markets are circled and the optimal matching of the three-sided market is shown in boldface. The payoff vector $u=(6,0 ; 0,8 ; 6,8)$ belongs to the core but cannot be obtained by core allocations of the three underlying two-sided assignment markets. Indeed, if there existed $\left(x^{1}, y^{1}\right) \in C\left(\gamma^{12}\right),\left(x^{2}, z^{2}\right) \in C\left(\gamma^{13}\right)$ and $\left(y^{3}, z^{3}\right) \in C\left(\gamma^{23}\right)$ such that $\left(x^{1}+x^{2} ; y^{1}+y^{3} ; z^{2}+z^{3}\right)=(6,0 ; 0,8 ; 6,8)$, then $0=x_{2}^{1}+x_{2}^{2}$ and $0=y_{1}^{1}+y_{1}^{3}$ imply $x_{2}^{2}=y_{1}^{3}=0$. Then, from the core constraints in the underlying two-sided markets, $x_{2}^{2}+z_{2}^{2}=5$ and $y_{1}^{3}+z_{1}^{3}=2$, we obtain $z_{2}^{2}=5$ and $z_{1}^{3}=2$. Now, $6=z_{1}^{2}+z_{1}^{3}$ implies $z_{1}^{2}=4$, and by substitution in $\left(x^{2}, z^{2}\right)$ we obtain $\left(x^{2}, z^{2}\right)=\left(x_{1}^{2}, 0 ; 4,5\right)$. But such a payoff vector is not in the core of $\gamma^{13}$ since the two core constraints $x_{1}^{2}+z_{1}^{2}=x_{1}^{2}+4=6$ and $x_{1}^{2}+z_{2}^{2}=x_{1}^{2}+5 \geq 9$ are not compatible.

Once established our model, and shown one subclass with non-empty core, we look for a notion of reduction that makes the core a consistent solution on the class of generalized three-sided assignment markets. 


\section{Consistency of the core and the nucleolus}

In this section, we introduce the derived market (and game) for the generalized three-sided assignment market, and the corresponding consistency property.

Given any coalitional game, and given a particular distribution of the worth of the grand coalition, we may ask what happens when some agents leave the market after being paid according to that given distribution. The remaining agents must reevaluate the worth of all the coalitions they can form. The different ways in which this reevaluation is done correspond to the different notions of reduced game that exist in the literature.

Maybe the best known notion of reduced game is that of Davis and Maschler (1965), where the remaining coalitions take into account what they could obtain by joining some agents that have left, with the condition of preserving the amount they have already been paid.

Definition 2 (Davis and Maschler, 1965) Given a generalized three-sided assignment game $\left(N, w_{\gamma}\right)$, a non-empty coalition $S$ and a payoff vector $x \in$ $\mathbb{R}^{N \backslash S}$, the Davis and Maschler reduced game for the coalition $S$ at $x$ is the game $\left(S, w_{\gamma}^{S, x}\right)$ that is defined by

$$
w_{\gamma}^{S, x}(T)= \begin{cases}0 & \text { if } T=\emptyset \\ w_{\gamma}(N)-x(N \backslash S) & \text { if } T=S, \\ \max _{Q \subseteq N \backslash S}\left\{w_{\gamma}(T \cup Q)-x(Q)\right\} & \text { otherwise. }\end{cases}
$$

In general, the reduced game of a generalized three-sided assignment game is not superadditive, and hence it is not a generalized three-sided assignment game. Take for instance coalition $S=\left\{1,2^{\prime}, 1^{\prime \prime}, 2^{\prime \prime}\right\}$ and the core element $u=(6,0 ; 0,8 ; 6,8)$ in the market of Example 3 and notice that $w_{\gamma}^{S, u}\left(\left\{1^{\prime \prime}\right\}\right)+$ $w_{\gamma}^{S, u}\left(\left\{2^{\prime \prime}\right\}\right)=3+5>7=w_{\gamma}^{S, u}\left(\left\{1^{\prime \prime}, 2^{\prime \prime}\right\}\right)$.

To solve this, we introduce a new reduction for the generalized three-sided assignment market (and game) that extends the derived game introduced by Owen (1992) for the two-sided case. We will see that this notion of reduced game is closely related to the Davis and Maschler reduction.

Definition 3 Given a generalized three-sided assignment market $\gamma=\left(M_{1}, M_{2}, M_{3} ; v\right)$, $\emptyset \neq S=S_{1} \cup S_{2} \cup S_{3}, S \neq N$, where $S_{1} \subseteq M_{1}, S_{2} \subseteq M_{2}, S_{3} \subseteq M_{3}$ and $x \in \mathbb{R}^{N \backslash S}$, the derived market at $S$ and $x$ is $\hat{\gamma}^{S, x}=\left(S_{1}, S_{2}, S_{3} ; \hat{v}^{S, x}\right)$ where

$$
\hat{v}^{S, x}(E)=\max _{\substack{Q \subseteq N \backslash S \\ E \cup Q \in \mathcal{B}}}\{v(E \cup Q)-x(Q)\} \text { for all } E \in \mathcal{B}^{S} .
$$

Then, the corresponding derived game at $S$ and $x$ is $\left(S, w_{\hat{\gamma}^{S, x}}\right)$ where for all $R \subseteq S$,

$$
w_{\hat{\gamma}^{S, x}}(R)=\max _{\mu \in \mathcal{M}\left(M_{1} \cap R, M_{2} \cap R, M_{3} \cap R\right)}\left\{\sum_{E \in \mu} \hat{v}^{S, x}(E)\right\} .
$$


To obtain the derived game, we first consider the valuation in the reduced situation of the basic coalitions of the submarket. The valuation of such a basic coalition is obtained by allowing it to cooperate only with agents that have left but with whom it can form a basic coalition of the initial market. In particular, when $E=\{i, j, k\}$ with $i \in S_{1}, j \in S_{2}$ and $k \in S_{3}$, then $\hat{v}^{S, x}(\{i, j, k\})=v(\{i, j, k\})$. Thus, the worth $w_{\hat{\gamma}^{S, x}}(R)$ in the derived game for any coalition $R \subseteq S$ is obtained from the valuations $\hat{v}^{S, x}$ of the basic coalitions in $\mathcal{B}^{S}$ by imposing superadditivity. Hence, the derived assignment game is always a superadditive game.

Notice that in (2) different basic coalitions $E$ in the same matching $\mu \in$ $\mathcal{M}\left(M_{1} \cap R, M_{2} \cap R, M_{3} \cap R\right)$ can use the same coalition $Q \subseteq N \backslash S$ to establish their value $\hat{v}^{S, x}(E)$. Thus, $\left(S, w_{\hat{\gamma}^{S, x}}\right)$ is not a game in the ordinary sense; it serves only to determine the distribution of $w_{\hat{\gamma} S, x}(S)$ among the members of $S$. Also in the Davis and Maschler reduced game the expectations of different disjoint subcoalitions may not be compatible with each other, because they may require cooperation of the same subset of $N \backslash S$.

However, it is interesting to remark that the worth of the grand coalition of the derived game (at a core allocation) is indeed attainable. The reason is there exists an optimal matching of the derived game such that no two basic coalitions of this matching need the cooperation of a same outside agent to attain their worth. We will argue this fact in Remark 1, below the proof of Theorem 1.

A market with some empty sector is a two-sided market (with individual reservation values) and the definition of derived game coincides with the one given by Owen (1992) for these markets.

Given a game $(N, w)$, its superadditive cover is the minimal superadditive game $(N, \tilde{w})$ such that $\tilde{w} \geq w$. Next proposition extends a result obtained for two-sided assignment games by Owen (1992). We show that for any generalized three-sided assignment game $\left(N, w_{\gamma}\right)$, its derived game $\left(S, w_{\hat{\gamma} S, x}\right)$ at any coalition $S$ and core allocation $x$ is the superadditive cover of the corresponding Davis and Maschler reduced game $\left(S, w_{\gamma}^{S, x}\right)$. This means that the derived game of a generalized three-sided assignment market is closely related to the Davis and Maschler reduced game. The proof is consigned to the Appendix.

Proposition 2 Let $\gamma=\left(M_{1}, M_{2}, M_{3} ; v\right)$ be a generalized three-sided assignment market, $N=M_{1} \cup M_{2} \cup M_{3},\left(N, w_{\gamma}\right)$ the associated generalized three-sided game and $x \in C(\gamma)$. Then for any $\emptyset \neq S \subsetneq N$, the derived game $\left(S, w_{\hat{\gamma}^{S, x}}\right)$, where $\hat{\gamma}^{S, x}=\left(M_{1} \cap S, M_{2} \cap S, M_{3} \cap S ; \hat{v}^{S, x}\right)$, is the superadditive cover of the Davis and Maschler reduced game $\left(S, w_{\gamma}^{S, x}\right)$.

Our objective now is to introduce a consistency property with respect to the derived market. We name this property derived consistency.

Before doing that, we need to introduce the notion of solution in the class of generalized three-sided assignment markets or games. Next definition extends to our setting the notion of feasibility that is usual in two-sided assignment markets. 
Definition 4 Given a generalized three-sided assignment market $\gamma=\left(M_{1}, M_{2}, M_{3} ; v\right)$, an allocation $x \in \mathbb{R}^{M_{1}} \times \mathbb{R}^{M_{2}} \times \mathbb{R}^{M_{3}}$ is feasible-by-matching if there exists a matching $\mu \in \mathcal{M}\left(M_{1}, M_{2}, M_{3}\right)$ such that for all $E \in \mu, x(E)=v(E)$.

In that case, we say that $x$ and $\mu$ are compatible. Notice that a matching $\mu$ compatible with $x$ may not be optimal. Moreover, the set of feasible-bymatching allocations is always non-empty since we can take the matching $\mu=\{\{i\}\}_{i \in N}$ and then $x=(v(\{i\}))_{i \in N}$ is feasible with respect to $\mu$.

Definition 5 A solution on a class $\Gamma \subseteq \Gamma_{3-G A M}$ is a correspondence $\sigma$ that assigns a subset of feasible-by-matching payoff vectors to each $\gamma \in \Gamma$.

Given $\gamma \in \Gamma$, we write $\sigma(\gamma)$ to denote the subset of feasible-by-matching payoff vectors assigned by solution $\sigma$ to the assignment market $\gamma$. Notice that a solution $\sigma$ is allowed to be empty. The core correspondence and the mapping that gives to each agent his/her individual value (compatible with the matching formed by all individual coalitions) are examples of solutions on the class of generalized three-sided assignment markets. Similarly, the nucleolus, which will be defined below, is a solution on the subclass of balanced generalized three-sided assignment markets.

Definition 6 A solution $\sigma$ on the class of generalized three-sided assignment markets satisfies derived consistency if for all $\gamma=\left(M_{1}, M_{2}, M_{3} ; v\right)$, all $\emptyset \neq$ $S \subsetneq N$ and all $x \in \sigma(\gamma)$, it holds $x_{\mid S} \in \sigma\left(\hat{\gamma}^{S, x}\right)$.

Next theorem shows that the core satisfies derived consistency on the domain of generalized three-sided assignment markets.

Theorem 1 On the domain of generalized three-sided assignment markets, the core satisfies derived consistency.

Proof Let $\gamma=\left(M_{1}, M_{2}, M_{3} ; v\right)$ be a generalized three-sided assignment market, let $x$ be a core allocation and $\emptyset \neq S \subsetneq M_{1} \cup M_{2} \cup M_{3}$. To simplify notation, let us write $\hat{v}=\hat{v}^{S, x}$ and $\hat{w}=w_{\hat{\gamma}^{S, x}}$.

Consider all possible basic coalitions in $\mathcal{B}^{S}$. First, for all $\{i, j, k\} \in M_{1} \cap$ $S \times M_{2} \cap S \times M_{3} \cap S, x_{i}+x_{j}+x_{k} \geq v(\{i, j, k\})=\hat{v}(\{i, j, k\})$. Secondly, for all $\{i, j\} \in\left(M_{1} \cap S\right) \times\left(M_{2} \cap S\right), x_{i}+x_{j} \geq v(\{i, j\})$ and $x_{i}+x_{j} \geq v(\{i, j, k\})-x_{k}$ for all $k \in M_{3} \backslash S$. Hence, $x_{i}+x_{j} \geq \hat{v}(\{i, j\})$. Finally, for all $i \in M_{1} \cap S$, $x_{i} \geq v(\{i\})$, and $x_{i} \geq v(\{i, j\})-x_{j}$ for all $j \in M_{2} \backslash S$, and $x_{i} \geq v(\{i, k\})-x_{k}$ for all $k \in M_{3} \backslash S$, and $x_{i} \geq v(\{i, j, k\})-x_{j}-x_{k}$ for all $j \in M_{2} \backslash S$ and for all $k \in M_{3} \backslash S$. Hence, $x_{i} \geq \hat{v}(\{i\})$. Proceeding similarly for the remaining $E \in \mathcal{B}^{S}$, we obtain

$$
x(E) \geq \hat{v}(E) \text { for all } E \in \mathcal{B}^{S} .
$$

Finally, it remains to show that $x(S)=\hat{w}(S)$. Expression (3) implies $x(R) \geq \hat{w}(R)$ for all $R \subseteq S$. Now, appyling Proposition 2 we obtain

$$
x(S) \geq \hat{w}(S) \geq w_{\gamma}^{S, x}(S)=x(S),
$$


where the second inequality follows from Proposition 2 and the last equality from the Davis and Maschler reduced game property of the core (see Peleg, 1986). Thus, $x(S)=\hat{w}(S)$ and this completes the proof of $x_{\mid S} \in C\left(\hat{\gamma}^{S, x}\right)$.

As a consequence of the proof of the above theorem, we can justify, by means of the following remark, that the worth of the grand coalition of the derived game is indeed attainable.

Remark 1 An optimal matching of the derived market at a core allocation is induced by an optimal matching of the initial market. To see that, take $\gamma=\left(M_{1}, M_{2}, M_{3} ; v\right)$ a balanced generalized three-sided assignment market and $\mu$ an optimal matching, $\mu \in \mathcal{M}_{\gamma}\left(M_{1}, M_{2}, M_{3}\right)$. Let $\hat{\gamma}^{S, x}=\left(M_{1} \cap S, M_{2} \cap\right.$ $\left.S, M_{3} \cap S ; \hat{v}^{S, x}\right)$ be the derived market at $S \subseteq M_{1} \cup M_{2} \cup M_{3}$ and $x \in C(\gamma)$. It turns out that $\mu_{\mid S}=\{E \cap S \mid E \in \mu\}$ is optimal for $\hat{\gamma}^{S, x}$. Indeed, given any other $\mu^{\prime} \in \mathcal{M}_{\hat{\gamma}^{S, x}}\left(M_{1} \cap S, M_{2} \cap S, M_{3} \cap S\right)$,

$$
\begin{aligned}
\sum_{E \in \mu^{\prime}} \hat{v}^{S, x}(E) & \leq \sum_{E \in \mu^{\prime}} x(E)=x(S)=\sum_{E \in \mu_{\mid S}} x(E) \\
& =\sum_{E \in \mu_{\mid S}} v(D(E))-x(D(E) \backslash E) \leq \sum_{E \in \mu_{\mid S}} \hat{v}^{S, x}(E),
\end{aligned}
$$

where the first inequality follows from (3); for all $E \in \mu_{\mid S}, D(E)$ is defined as the unique basic coalition in $\mu$ such that $D(E) \cap S=E$; and the last inequality follows from (1). Hence, $\mu_{\mid S}$ is optimal for $\hat{\gamma}^{S, x}$. Because of that, no two basic coalitions of $\mu_{\mid S}$ need the cooperation of the same outside agent to attain their worth.

To finish this section we show another solution concept that satisfies derived consistency. The nucleolus is a well-known single-valued solution for coalitional games introduced by Schmeidler (1969). When the game is balanced, the nucleolus is the unique core allocation that lexicographically minimizes the vector of decreasingly-ordered excesses of coalitions. ${ }^{2}$

The nucleolus of a generalized three-sided assignment market $\gamma=\left(M_{1}, M_{2}, M_{3} ; v\right)$ is the nucleolus of the associated assignment game $\left(N, w_{\gamma}\right)$, and it will be denoted by $\eta(\gamma)$. Next, we show that when a generalized three-sided assignment market is balanced the nucleolus also satisfies derived consistency.

Theorem 2 On the class of balanced generalized three-sided assignment markets, the nucleolus satisfies derived consistency.

Proof Let $\gamma=\left(M_{1}, M_{2}, M_{3} ; v\right)$ be a balanced generalized three-sided assignment market, $\eta(\gamma)=\eta$ be the nucleolus and $\emptyset \neq S \subsetneq M_{1} \cup M_{2} \cup M_{3}$. Since the nucleolus satisfies the Davis and Maschler reduced game property (Potters, 1991), $\eta_{\mid S}=\eta\left(w_{\gamma}^{S, \eta}\right)$ which implies $\eta(S)=w_{\gamma}^{S, \eta}(S)$. On the other hand,

\footnotetext{
${ }^{2}$ Given a game $(N, r)$, the excess of a coalition $S \subseteq N$ at a payoff vector $x \in \mathbb{R}^{N}$ is $r(S)-\sum_{i \in S} x_{i}$
} 
since $\eta \in C(\gamma)$, by Theorem 1 we know that $\eta_{\mid S} \in C\left(w_{\hat{\gamma}^{S, \eta}}\right)$ which implies $\eta(S)=w_{\hat{\gamma}^{S, \eta}}(S)$. Hence, taking into account Proposition 2, we have that the Davis and Maschler reduced game $\left(S, w_{\gamma}^{S, \eta}\right)$ and its superadditive cover have the same efficiency level, which implies (Miquel and Núñez, 2011) that both games have the same nucleolus. Therefore, $\eta_{\mid S}=\eta\left(w_{\hat{\gamma}^{S, \eta}}\right)$.

In the next section we combine derived consistency with two additional properties in order to characterize the core of generalized three-sided assignment games.

\section{An axiomatic characterization of the core}

In this section, we give an axiomatic characterization of the core on the class of generalized three-sided assignment markets making use of derived consistency and two additional properties, singleness best and individual antimonotonicity, that are introduced in the sequel.

Definition 7 A solution $\sigma$ on $\Gamma \subseteq \Gamma_{3-G A M}$ satisfies singleness best if for all $\gamma=\left(M_{1}, M_{2}, M_{3} ; v\right) \in \Gamma$, it holds that whenever the partition in singletons is optimal in $\gamma$, then $(v(\{i\}))_{i \in N} \in \sigma(\gamma)$.

Singleness best simply says that if the partition in individual coalitions is optimal, then the vector of individual values should be an outcome of the solution. This axiom has some resemblance with the zero inessential game property of Hwang and Sudhölter (2001) in the sense that it is a non-emptiness axiom for generalized three-sided assignment games that are trivial or inessential.

Before introducing the property of individual anti-monotonicity we need to establish how to compare the individual values of all agents across different games.

Given two payoff vectors $x=\left(x_{i}\right)_{i \in N}, x^{\prime}=\left(x_{i}^{\prime}\right)_{i \in N}$ in $\mathbb{R}^{N}$ and $\mu \in$ $\mathcal{M}\left(M_{1}, M_{2}, M_{3}\right)$, we write $x^{\prime} \geq_{\mu} x$ when $x_{i}=x_{i}^{\prime}$ for all $\{i\} \in \mu$ and $x_{i}^{\prime} \geq x_{i}$ if $\{i\} \notin \mu$. That is, $x^{\prime}$ is greater than $x$ with respect to $\mu$ when agents that are matched with some other partner receive at least as much in $x^{\prime}$ than in $x$, while agents that are alone receive the same payoff in both allocations.

Definition 8 A solution $\sigma$ on $\Gamma \subseteq \Gamma_{3-G A M}$ satisfies individual anti-monotonicity if for all $\gamma^{\prime}=\left(M_{1}, M_{2}, M_{3} ; v^{\prime}\right) \in \Gamma$, all $\gamma=\left(M_{1}, M_{2}, M_{3} ; v\right) \in \Gamma$, all $u \in \sigma\left(\gamma^{\prime}\right)$ and matching $\mu$ compatible with $u$, if $v(E)=v^{\prime}(E)$ for all $E \in \mathcal{B}$ with $|E|>1$ and $\left(v^{\prime}(\{i\})\right)_{i \in N} \geq_{\mu}(v(\{i\}))_{i \in N}$, then it holds $u \in \sigma(\gamma)$.

Individual anti-monotonicity says that if the individual values decrease (in the sense defined above) any payoff vector in the solution of the original market should remain in the solution of the new market. Notice that the value of pairs and triplets coincide in both markets. Individual anti-monotonicity is a weaker version of anti-monotonicity introduced by Keiding (1986) and also used by Toda (2003). 
Now, we characterize the core on the class of generalized three-sided assignment games by means of derived consistency, singleness best and individual anti-monotonicity.

Theorem 3 On the domain of generalized three-sided assignment markets, the core is the unique solution that satisfies derived consistency, singleness best and individual anti-monotonicity.

Proof By Theorem 1 we know the core satisfies derived consistency. It is straightforward that the core satisfies singleness best and individual antimonotonicity. Assume now that $\sigma$ is a solution on $\Gamma_{3-G A M}$ also satisfying these axioms. Take any $\gamma=\left(M_{1}, M_{2}, M_{3} ; v\right) \in \Gamma_{3-G A M}$.

We first show that $\sigma(\gamma) \subseteq C(\gamma)$. Take $x \in \sigma(\gamma)$. We need to show that $x$ satisfies coalitional rationality and efficiency. Notice that if some side of the market is empty, the game is a two-sided assignment market and the statement follows from Proposition 2 in (Llerena et al, 2015). So, we can assume without loss of generality that $M_{l} \neq \emptyset$ for all $l \in\{1,2,3\}$. Then, for all $i \in M_{1} \cup M_{2} \cup M_{3}$ consider the derived market relative to $S=\{i\}$ at $x$. By derived consistency of $\sigma, x_{i} \in \sigma\left(\hat{\gamma}^{\{i\}, x}\right)$. Moreover, feasibility-bymatching of $\sigma$ implies that $x_{i}=\hat{v}^{\{i\}, x}(\{i\})$. Now, let $E \in \mathcal{B}$ be any basic coalition such that $i \in E$. By definition of derived market at $\{i\}$ and $x$ we have $x_{i}=\hat{v}^{\{i\}, x}(\{i\}) \geq v(E)-\sum_{k \in E \backslash\{i\}} x_{k}$. Hence, $\sum_{k \in E} x_{k} \geq v(E)$ which states that $x$ satisfies coalitional rationality.

In order to prove efficiency, let $\mu$ be an optimal matching and $\mu^{\prime}$ be a matching compatible with $x$. Then, $w_{\gamma}(N)=\sum_{E \in \mu} v(E) \leq \sum_{E \in \mu}\left(\sum_{i \in E} x_{i}\right)=$ $\sum_{E \in \mu^{\prime}}\left(\sum_{i \in E} x_{i}\right)=\sum_{E \in \mu^{\prime}} v(E)$, where the last equality follows from the fact that $\mu^{\prime}$ is compatible with $x$. Since $\mu$ is optimal and $w_{\gamma}(N) \leq \sum_{E \in \mu^{\prime}} v(E)$, we get that $\mu^{\prime}$ is also optimal and $x$ is efficient. Hence, $x \in C(\gamma)$ and we have proved $\sigma(\gamma) \subseteq C(\gamma)$

To show that $C(\gamma) \subseteq \sigma(\gamma)$, take $u \in C(\gamma)$ and $\mu \in \mathcal{M}\left(M_{1}, M_{2}, M_{3}\right)$ compatible with $u$. Then, $\mu$ is optimal for $\gamma$. Now, define a market $\gamma^{\prime}=$ $\left(M_{1}, M_{2}, M_{3} ; v^{\prime}\right)$ where $v^{\prime}(E)=v(E)$ for all $E \in \mathcal{B}$ such that $|E|>1$ and $v^{\prime}(E)=u_{i}$ for all $E=\{i\}$. Notice that $v^{\prime}(\{i\})=u_{i}=v(\{i\})$ for all $\{i\} \in \mu$ and $v^{\prime}(\{i\})=u_{i} \geq v(\{i\})$ for all $\{i\} \notin \mu$. Hence, $\left(v^{\prime}(\{i\})\right)_{i \in N} \geq_{\mu}(v(\{i\}))_{i \in N}$. Let us see that $\mu^{\prime}=\{\{i\} \mid i \in N\}$ is optimal for $\gamma^{\prime}$. To this end, take any matching $\mu^{\prime \prime} \in \mathcal{M}\left(M_{1}, M_{2}, M_{3}\right)$. Then,

$$
\begin{aligned}
\sum_{E \in \mu^{\prime}} v^{\prime}(E) & =\sum_{i \in N} v^{\prime}(\{i\})=\sum_{i \in N} u_{i}=\sum_{\substack{E \in \mu^{\prime \prime} \\
|E|>1}} \sum_{\substack{\mid \in E\\
}} u_{i}+\sum_{\substack{E \in \mu^{\prime \prime} \\
|E|=1}} \sum_{i \in E} u_{i} \\
& \geq \sum_{\substack{E \in \mu^{\prime \prime} \\
|E|>1}} v^{\prime}(E)+\sum_{\substack{E \in \mu^{\prime \prime} \\
|E|=1}} v^{\prime}(E)=\sum_{E \in \mu^{\prime \prime}} v^{\prime}(E) .
\end{aligned}
$$


The inequality follows from the fact that $u \in C(\gamma)$ and the relationship between $v$ and $v^{\prime}$. Thus, $\mu^{\prime}$ is optimal for $\gamma^{\prime}$. By singleness best, $u=\left(u_{i}\right)_{i \in N}=$ $\left(v^{\prime}(\{i\})\right)_{i \in N} \in \sigma\left(\gamma^{\prime}\right)$ and then, by individual anti-monotonicity, $u \in \sigma(\gamma)$. Hence, $C(\gamma) \subseteq \sigma(\gamma)$. Together with the reverse inclusion, $\sigma(\gamma) \subseteq C(\gamma)$, we conclude that $C(\gamma)=\sigma(\gamma)$.

We now show that no axiom in the above characterization is implied by the others. To this end, we introduce different solutions satisfying all axioms but one.

Example 4 For all $\gamma=\left(M_{1}, M_{2}, M_{3} ; v\right) \in \Gamma_{3-G A M}$, let us consider

$$
\sigma_{1}(\gamma)=\emptyset
$$

Clearly, $\sigma_{1}$ satisfies derived consistency and individual anti-monotonicity but not singleness best.

Example 5 For all $\gamma=\left(M_{1}, M_{2}, M_{3} ; v\right) \in \Gamma_{3-G A M}$, write $N=M_{1} \cup M_{2} \cup M_{3}$ and let us consider

$$
\sigma_{2}(\gamma)=\left\{\begin{array}{l|l}
u \in \mathbb{R}^{N} & \begin{array}{l}
u \text { is feasible-by-matching for } \gamma \\
u_{i} \geq w_{\gamma}(\{i\}), \text { for all } i \in N, \\
u(N)=w_{\gamma}(N)
\end{array}
\end{array}\right\}
$$

Notice that if $u \in \sigma_{2}(\gamma)$, every matching $\mu$ that is compatible with $u$ is optimal. It can be easily checked that $\sigma_{2}$ satisfies singleness best and individual antimonotonicity but, since $\sigma_{2}$ is different from the core, the characterization of the core in Theorem 3 implies that $\sigma_{2}$ does not satisfy derived consistency.

Example 6 For all $\gamma=\left(M_{1}, M_{2}, M_{3} ; v\right) \in \Gamma_{3-G A M}$, let $\eta(\gamma)$ be the nucleolus of $\gamma$ and consider

$$
\sigma_{3}(\gamma)= \begin{cases}\emptyset & \text { if } C(\gamma)=\emptyset \\ \{\eta(\gamma)\} & \text { if } C(\gamma) \neq \emptyset\end{cases}
$$

The solution $\sigma_{3}$ satisfies singleness best and derived consistency (see Theorem 2 ), but, since $\sigma_{3}$ is different from the core, the characterization of the core in Theorem 3 implies that $\sigma_{3}$ does not satisfy individual anti-monotonicity.

These three examples prove that none of the axioms is redundant in the above characterization of the core.

\section{Core and competitive equilibria}

We now focus on the particular case where $M_{1}=\{1, \ldots, m\}$ and $M_{2}=$ $\left\{1^{\prime}, \ldots, m^{\prime}\right\}$ are two sets of sellers, each selling an indivisible good. Goods of sellers in $M_{1}$ are of a different type of those of sellers in $M_{2}$. The third sector $M_{3}=\left\{1^{\prime \prime}, \ldots, m^{\prime \prime}\right\}$ is formed by buyers, each interested in buying at most one unit of each type of good. Each seller $r \in M_{1} \cup M_{2}$ has a reservation value 
$c_{r} \geq 0$ for his object, meaning he will not sell for a price lower than that. We denote by $c$ the vector of sellers' reservation values.

We denote by $\mathcal{B}^{k}$, the set of basic coalitions that contain buyer $k \in M_{3}$, $\mathcal{B}^{k}=\{E \in \mathcal{B} \mid k \in E\}$. Then, each buyer $k \in M_{3}$ places a value $w^{k}(E) \in \mathbb{R}_{+}$ on each basic coalition $E \in \mathcal{B}^{k}$ and we denote by $w=\left(w^{k}\right)_{k \in M_{3}}$ the vector of buyers' valuations.

All these valuations $(w, c)$ give rise to a generalized three-sided assignment market $\left(M_{1}, M_{2}, M_{3} ; v^{w, c}\right)$ where $v^{w, c}(E)=w^{k}(E)-c(E \backslash\{k\})$ if $E \in \mathcal{B}^{k}$ for some $k \in M_{3}$ and $v^{w, c}(E)=0$ if $E \in \mathcal{B}$ with $E \cap M_{3}=\emptyset$. We denote by $\Gamma_{S S B}$ this subclass of generalized three-sided assignment markets that are defined by some valuations $(w, c)$.

We want to show that each core allocation is the result of trading goods following an optimal matching and according to some prices. To introduce the notion of competitive price vector, some previous definitions are needed.

Given a generalized three-sided assignment market $\gamma=\left(M_{1}, M_{2}, M_{3} ; v^{w, c}\right) \in$ $\Gamma_{S S B}$, a feasible price vector is $p \in \mathbb{R}_{+}^{M_{1} \cup M_{2}}$ such that $p_{r} \geq c_{r}$ for all $r \in$ $M_{1} \cup M_{2}$.

Next, for each feasible price vector $p \in \mathbb{R}_{+}^{M_{1} \cup M_{2}}$ we introduce the demand set of each buyer in sector $M_{3}$.

Definition 9 Given a market $\gamma=\left(M_{1}, M_{2}, M_{3} ; v^{w, c}\right) \in \Gamma_{S S B}$, the demand set of a buyer $k \in M_{3}$ at a feasible price vector $p \in \mathbb{R}_{+}^{M_{1} \cup M_{2}}$ is

$D_{k}(p)=\left\{E \in \mathcal{B}^{k} \mid w^{k}(E)-p(E \backslash\{k\}) \geq w^{k}\left(E^{\prime}\right)-p\left(E^{\prime} \backslash\{k\}\right)\right.$ for all $\left.E^{\prime} \in \mathcal{B}^{k}\right\}$.

Note that $D_{k}(p)$ describes the set of basic coalitions containing buyer $k$ that maximize the net valuation of buyer $k$ at prices $p$. Notice also that the demand set of a buyer $k \in M_{3}$ is always non-empty. If $\mu \in \mathcal{M}\left(M_{1}, M_{2}, M_{3}\right)$, for all $k \in M_{3}$ we will write $\mu(k)$ to denote the basic coalition $E$ such that $k \in E \in \mu$.

Given a matching $\mu \in \mathcal{M}\left(M_{1}, M_{2}, M_{3}\right)$, we say a seller $r \in M_{1} \cup M_{2}$ is unassigned (by $\mu$ ) if there is no $k \in M_{3}$ such that $r \in \mu(k)$

Now, we can introduce the notion of competitive equilibrium for our market.

Definition 10 Given a market $\gamma=\left(M_{1}, M_{2}, M_{3} ; v^{w, c}\right) \in \Gamma_{S S B}$, a pair $(p, \mu)$, where $p \in \mathbb{R}_{+}^{M_{1} \cup M_{2}}$ is a feasible price vector and $\mu \in \mathcal{M}\left(M_{1}, M_{2}, M_{3}\right)$, is a competitive equilibrium if

i for all buyer $k \in M_{3}, \mu(k) \in D_{k}(p)$,

ii for all seller $r \in M_{1} \cup M_{2}$, if $r$ is unassigned by $\mu$, then $p_{r}=c_{r}$.

If a pair $(p, \mu)$ is a competitive equilibrium, then we say that the price vector $p$ is a competitive equilibrium price vector. The corresponding payoff vector for a given pair $(p, \mu)$ is called competitive equilibrium payoff vector. This payoff vector is $(x(p, \mu), y(p, \mu), z(p, \mu)) \in \mathbb{R}^{M_{1}} \times \mathbb{R}^{M_{2}} \times \mathbb{R}^{M_{3}}$, defined by

$$
\begin{aligned}
& x_{i}(p, \mu)=p_{i}-c_{i} \text { for all sellers } i \in M_{1}, \\
& y_{j}(p, \mu)=p_{j}-c_{j} \text { for all sellers } j \in M_{2}, \\
& z_{k}(p, \mu)=w^{k}(\mu(k))-p(\mu(k) \backslash\{k\}) \text { for all buyers } k \in M_{3} .
\end{aligned}
$$


We denote the set of competitive equilibrium payoff vectors of market $\gamma$ by $\mathcal{C E}(\gamma)$.

We now study the relationship between the core of the market $\gamma=\left(M_{1}, M_{2}, M_{3} ; v^{w, c}\right) \in$ $\Gamma_{S S B}$ and the set of competitive equilibrium payoff vectors. First, we need to point out that if a matching $\mu$ constitutes a competitive equilibrium with a feasible price vector $p$, then $\mu$ is an optimal matching. The proof is consigned to the Appendix.

Lemma 1 Given a market $\gamma=\left(M_{1}, M_{2}, M_{3} ; v^{w, c}\right) \in \Gamma_{S S B}$, if a pair $(p, \mu)$ is a competitive equilibrium, then $\mu$ is an optimal matching.

Now, we can give the main result in this section.

Theorem 4 Given a market $\gamma=\left(M_{1}, M_{2}, M_{3} ; v^{w, c}\right) \in \Gamma_{S S B}$, the core of the market, $C(\gamma)$, coincides with the set of competitive equilibrium payoff vectors, $\mathcal{C E}(\gamma)$

Proof First, we show that if $(p, \mu)$ is a competitive equilibrium, then its corresponding competitive equilibrium payoff vector $X=(x(p, \mu), y(p, \mu), z(p, \mu)) \in$ $\mathcal{C E}(\gamma)$ is a core element. Recall from its definition that $x_{i}(p, \mu)=p_{i}-c_{i}$ for all $i \in$ $M_{1}, y_{j}(p, \mu)=p_{j}-c_{j}$ for all $j \in M_{2}$ and $z_{k}(p, \mu)=w^{k}(\mu(k))-p(\mu(k) \backslash$ $\{k\})$ for all $k \in M_{3}$. Let us check that for all basic coalitions $E \in \mathcal{B}$ it holds $X(E) \geq v^{w, c}(E)$. Notice that if $E$ does not contain any buyer $k \in M_{3}$, then $v^{w, c}(E)=0$ and hence the core inequality holds. Otherwise, take $E \in \mathcal{B}$ such that $k \in E$ for some $k \in M_{3}$. Then,

$$
\begin{aligned}
X(E) & =p(E \backslash\{k\})-c(E \backslash\{k\})+w^{k}(\mu(k))-p(\mu(k) \backslash\{k\}) \\
& \geq p(E \backslash\{k\})-c(E \backslash\{k\})+w^{k}(E)-p(E \backslash\{k\}) \\
& =w^{k}(E)-c(E \backslash\{k\})=v^{w, c}(E),
\end{aligned}
$$

where the inequality follows from the fact that $(p, \mu)$ is a competitive equilibrium. It remains to check that $X$ is efficient. Since $\mu$ is a partition of $N=M_{1} \cup M_{2} \cup M_{3}$, we get

$$
\begin{aligned}
X(N) & =\sum_{k \in M_{3}}\left[w^{k}(\mu(k))-p(\mu(k) \backslash\{k\})\right]+p\left(M_{1} \cup M_{2}\right)-c\left(M_{1} \cup M_{2}\right) \\
& =\sum_{k \in M_{3}}\left[w^{k}(\mu(k))-p(\mu(k) \backslash\{k\})+p(\mu(k) \backslash\{k\})-c(\mu(k) \backslash\{k\})\right]+\sum_{l \notin \bigcup_{k \in M_{3}} \mu(k)}\left(p_{l}-c_{l}\right) \\
& =\sum_{k \in M_{3}}\left[w^{k}(\mu(k))-c(\mu(k) \backslash\{k\})\right] \\
& =\sum_{k \in M_{3}} v^{w, c}(\mu(k))=\sum_{E \in \mu} v^{w, c}(E),
\end{aligned}
$$

where the third equality holds since $p_{l}=c_{l}$ for unassigned objects $l$.

We have shown that if $(p, \mu)$ is a competitive equilibrium, then its competitive equilibrium payoff vector $X$ is a core allocation. 
Next, we show that the reverse implication holds. That is, if $X \in \mathbb{R}^{N}$ is a core allocation, then it is the payoff vector related to a competitive equilibrium $(p, \mu)$, where $\mu$ is any optimal matching and $p$ is a competitive equilibrium price vector.

Let us define $p \in \mathbb{R}^{M_{1}} \times \mathbb{R}^{M_{2}}$ by $p_{l}=X_{l}+c_{l}$ for all $l \in M_{1} \cup M_{2}$. Notice first that since $X \in C(\gamma)$, if an object $l \in M_{1} \cup M_{2}$ is unassigned by the matching $\mu$, then $p_{l}=X_{l}+c_{l}=c_{l}$. Moreover, $X(\mu(k))=v^{w, c}(\mu(k))$ for all $k \in M_{3}$ and $X\left(E^{\prime}\right) \geq v^{w, c}\left(E^{\prime}\right)$ for all $E^{\prime} \in \mathcal{B}^{k}$ where $k \in M_{3}$. Then, for all $k \in M_{3}$ and all $E^{\prime} \in \mathcal{B}^{k}$,

$$
\begin{aligned}
w^{k}(\mu(k))-p(\mu(k) \backslash\{k\}) & =v^{w, c}(\mu(k))+c(\mu(k) \backslash\{k\})-p(\mu(k) \backslash\{k\}) \\
& =X(\mu(k))+c(\mu(k) \backslash\{k\})-p(\mu(k) \backslash\{k\}) \\
& =X_{k} \\
& \geq v^{w, c}\left(E^{\prime}\right)-X\left(E^{\prime} \backslash\{k\}\right) \\
& =v^{w, c}\left(E^{\prime}\right)-\left[p\left(E^{\prime} \backslash\{k\}\right)-c\left(E^{\prime} \backslash\{k\}\right)\right] \\
& =w^{k}\left(E^{\prime}\right)-p\left(E^{\prime} \backslash\{k\}\right)
\end{aligned}
$$

where the inequality follows from the fact that $X \in C(\gamma)$. This shows that $\mu(k) \in D_{k}(p)$ which concludes the proof.

Once shown that on the class of generalized three-sided assignment markets the set of competitive equilibrium payoff vectors, $\mathcal{C E}(\gamma)$, coincides with the core of the market, $C(\gamma)$, we have that competitive equilibria exist for this model if and only if the core is non-empty. ${ }^{3}$

Notice to conclude that the class $\Gamma_{S S B}$ contains all classical three-sided assignment markets as defined in Kaneko and Wooders (1982) or Quint (1991). Indeed, the class $\Gamma_{S S B}$ is characterized by two facts: a) individual values are null, $v(\{i\})=0$ for all seller $i \in M_{1} \cup M_{2}$ and b) any pair of sellers is also valued at zero, $v(\{i, j\})=0$ if $i \in M_{1}$ and $j \in M_{2}$. Now, if we have any classical three-sided assignment market defined by a three-dimensional matrix $A=\left(a_{i j k}\right)_{(i, j, k) \in M_{1} \times M_{2} \times M_{3}}$, simply define $c_{i}=0$ for all $i \in M_{1}, c_{j}=0$ for all $j \in M_{2}$ and, for all $k \in M_{3}, w^{k}(\{i, j, k\})=a_{i j k}$ for all $(i, j) \in M_{1} \times M_{2}$, $w^{k}(\{i, k\})=0$ for all $i \in M_{1}, w^{k}(\{j, k\})=0$ for all $j \in M_{2}$. This defines a market in $\Gamma_{S S B}$.

Since $\Gamma_{S S B}$ contains all classical three-sided assignment markets, balancedness is not guaranteed in this class.

\section{Appendix}

Proof of Proposition 2:

\footnotetext{
${ }^{3}$ See Quint (1991a) for a characterization of the non-emptiness of the core of games in partition form in terms of the solutions of the linear program that provides an optimal matching.
} 
Proof Let us write $\hat{w}=w_{\hat{\gamma} S, x}$. We have to show that $\hat{w}$ is superadditive, $\hat{w} \geq w_{\gamma}^{S, x}$ and $\hat{w}$ is minimal with these two properties.

By definition, $\hat{w}$ is superadditive. Now, we show that $\hat{w}(T) \geq w_{\gamma}^{S, x}(T)$ for all $T \subseteq S$. Notice that, for all $T \subseteq S$ there exists $Q \subseteq N \backslash S$ such that

$$
w_{\gamma}^{S, x}(T)=w_{\gamma}(T \cup Q)-\sum_{l \in Q} x_{l} .
$$

Let $\mu$ be a matching on $T \cup Q$ such that $w_{\gamma}(T \cup Q)=\sum_{E \in \mu} v(E)$. We introduce the following partition of the set of basic coalitions in $\mu$ :

$I_{1}=\{\{i, j, k\} \in \mu \mid i \in T, j \in T, k \in T\}$

$I_{2}=\{\{i, j, k\} \in \mu \mid i \notin T, j \notin T, k \notin T\}$

$I_{3}=\{\{i, j, k\} \in \mu \mid i \in T, j \in T, k \notin T\}$

$I_{4}=\{\{i, j, k\} \in \mu \mid i \in T, j \notin T, k \notin T\}$

$I_{5}=\{\{i, j\} \in \mu \mid i \in T, j \in T\}$

$I_{6}=\{\{i, j\} \in \mu \mid i \notin T, j \notin T\}$

$I_{7}=\{\{i, j\} \in \mu \mid i \in T, j \notin T\}$

$I_{8}=\{\{i\} \in \mu \mid i \in T\}$.

$I_{9}=\{\{i\} \in \mu \mid i \notin T\}$.

We write $w_{\gamma}(T \cup Q)$ in terms of the above partition.

$$
\begin{aligned}
w_{\gamma}(T \cup Q) & =\sum_{\{i, j, k\} \in I_{1}} v(\{i, j, k\})+\sum_{\{i, j, k\} \in I_{2}} v(\{i, j, k\})+\sum_{\{i, j, k\} \in I_{3}} v(\{i, j, k\}) \\
& +\sum_{\{i, j, k\} \in I_{4}} v(\{i, j, k\})+\sum_{\{i, j\} \in I_{5}} v(\{i, j\})+\sum_{\{i, j\} \in I_{6}} v(\{i, j\}) \\
& +\sum_{\{i, j\} \in I_{7}} v(\{i, j\})+\sum_{\{i\} \in I_{8}} v(\{i\})+\sum_{\{i\} \in I_{9}} v(\{i\}) .
\end{aligned}
$$

Then, substitute (5) in equation (4) and distribute $\sum_{l \in Q} x_{l}$ among the sets of the partition.

$$
\begin{aligned}
w_{\gamma}^{S, x}(T) & =w_{\gamma}(T \cup Q)-\sum_{i \in Q} x_{i} \\
& =\sum_{\{i, j, k\} \in I_{1}} v(\{i, j, k\})+\sum_{\{i, j, k\} \in I_{2}} v(\{i, j, k\})-x_{i}-x_{j}-x_{k} \\
& +\sum_{\{i, j, k\} \in I_{3}} v(\{i, j, k\})-x_{k}+\sum_{\{i, j, k\} \in I_{4}} v(\{i, j, k\})-x_{j}-x_{k} \\
& +\sum_{\{i, j\} \in I_{5}} v(\{i, j\})+\sum_{\{i, j\} \in I_{6}} v(\{i, j\})-x_{i}-x_{j}+\sum_{\{i, j\} \in I_{7}} v(\{i, j\})-x_{j} \\
& +\sum_{\{i\} \in I_{8}} v(\{i\})+\sum_{\{i\} \in I_{9}} v(\{i\})-x_{i} .
\end{aligned}
$$

Since $x \in C(\gamma)$, the second, the sixth and the last term are non-positive.

Let us consider $\hat{v}=\hat{v}^{S, x}$ (see Definition 3). For all $t, r, s \in\{1,2,3\}$ such that $r \neq s$, $r \neq t, s \neq t$ and all $i \in M_{r} \cap T, j \in M_{s} \cap T$,

$$
\hat{v}(\{i, j\})=\max _{k \in Q \cap M_{t}}\left\{v(\{i, j, k\})-x_{k}, v(\{i, j\})\right\} .
$$

As a consequence, for all $\{i, j, k\} \in I_{3}, v(\{i, j, k\})-x_{k} \leq \hat{v}(\{i, j\})$ and for all $\{i, j\} \in I_{5}$, $v(\{i, j\}) \leq \hat{v}(\{i, j\})$.

Also, for all $t \in\{1,2,3\}$ and $l \in M_{t} \cap T$, if $r, s$ are such that $r \neq s, s \neq t$ and $t \neq r$, then, 
$\hat{v}(\{l\})=\max _{\substack{i \in M_{r} \cap Q \\ j \in M_{s} \cap Q}}\left\{v(\{i, j, l\})-x_{i}-x_{j}, v(\{i, l\})-x_{i}, v(\{j, l\})-x_{j}, v(\{l\})\right\}$.

As a consequence, for all $\{i, j, k\} \in I_{4}, v(\{i, j, k\})-x_{j}-x_{k} \leq \hat{v}(\{i\})$; for all $\{i, j\} \in I_{7}$, $v(\{i, j\})-x_{j} \leq \hat{v}(\{i\})$ and trivially $v(\{i\}) \leq \hat{v}(\{i\})$ for all $\{i\} \in I_{8}$.

To sum up, taking into account that $\hat{w}$ is superadditive by definition,

$$
w_{\gamma}^{S, x}(T) \leq \sum_{\{i, j, k\} \in I_{1}} \hat{v}(\{i, j, k\})+\sum_{\substack{\{i, j, k\} \in I_{3} \\\{i, j\} \in I_{5}}} \hat{v}(\{i, j\})+\sum_{\substack{\{i, j, k\} \in I_{4} \\\{i, j\} \in I_{7} \\\{i\} \in I_{8}}} \hat{v}(\{i\}) \leq \hat{w}(T)
$$

Now, we only need to show that $\hat{w}$ is the minimal superadditive game satisfying the above inequality. First, consider $\{k\} \in \mathcal{B}^{S}$. Then,

$$
\begin{aligned}
& w_{\gamma}^{S, x}(\{k\})=\max _{Q \subseteq N \backslash S}\left\{w_{\gamma}(\{k\} \cup Q)-x(Q)\right\} \\
& \geq \max _{Q \subseteq N \backslash S}\left\{w_{\gamma}(\{k\} \cup Q)-x(Q)\right\} \\
& \{k\} \cup Q \in \mathcal{B} \\
& \geq \max _{\substack{Q \subseteq N \backslash S \\
\{k\} \cup Q \in \mathcal{B}}}\{v(\{k\} \cup Q)-x(Q)\} \\
& =\hat{v}(\{k\}) \text {. }
\end{aligned}
$$

Similarly, we obtain

$$
\begin{aligned}
w_{\gamma}^{S, x}\{i, j\} & \geq \hat{v}(\{i, j\}) \text { for all }\{i, j\} \in \mathcal{B}^{S}, \\
w_{\gamma}^{S, x}(\{i, j, k\}) & \geq \hat{v}(\{i, j, k\}) \text { for all }\{i, j, k\} \in \mathcal{B}^{S} .
\end{aligned}
$$

Assume now $(N, w)$ is superadditive and $w \geq w_{\gamma}^{S, x}$. For all $T \subseteq S$, let $\mu$ be an optimal matching for $\hat{\gamma}_{\mid T}^{S, x}$. Then,

$$
\begin{aligned}
w(T) & \geq \sum_{\{i, j, k\} \in \mu} w(\{i, j, k\})+\sum_{\{i, j\} \in \mu} w(\{i, j\})+\sum_{\{k\} \in \mu} w(\{k\}) \\
& \geq \sum_{\{i, j, k\} \in \mu} w_{\gamma}^{S, x}(\{i, j, k\})+\sum_{\{i, j\} \in \mu} w_{\gamma}^{S, x}(\{i, j\})+\sum_{\{k\} \in \mu} w_{\gamma}^{S, x}(\{k\}) \\
& \geq \sum_{\{i, j, k\} \in \mu} \hat{v}(\{i, j, k\})+\sum_{\{i, j\} \in \mu} \hat{v}(\{i, j\})+\sum_{\{k\} \in \mu} \hat{v}(\{k\}) \\
& =\hat{w}(T),
\end{aligned}
$$

where the last inequality follows from (6), (7) and (8).

This shows that $\hat{w}$ is the minimal superadditive game such that $\hat{w} \geq w_{\gamma}^{S, x}$, which implies that $\hat{w}$ is the superadditive cover of $w_{\gamma}^{S, x}$.

Proof of Lemma 1:

Proof In order to see this, we need to show that if $(p, \mu)$ is a competitive equilibrium, then the matching $\mu$ is a partition of maximal value. Consider a competitive equilibrium $(p, \mu)$ 
and another matching $\mu^{\prime} \in \mathcal{M}\left(M_{1}, M_{2}, M_{3}\right)$. Then,

$$
\begin{aligned}
& \sum_{E \in \mu} v^{w, c}(E)=\sum_{k \in M_{3}} w^{k}(\mu(k))-c(\mu(k) \backslash\{k\}) \\
& \geq \sum_{k \in M_{3}} w^{k}\left(\mu^{\prime}(k)\right)-c(\mu(k) \backslash\{k\})-p\left(\mu^{\prime}(k) \backslash\{k\}\right)+p(\mu(k) \backslash\{k\}) \\
& =\sum_{k \in M_{3}} w^{k}\left(\mu^{\prime}(k)\right)-c(\mu(k) \backslash\{k\})-p\left(\bigcup_{k \in M_{3}} \mu^{\prime}(k) \backslash M_{3}\right)+p\left(\bigcup_{k \in M_{3}} \mu(k) \backslash M_{3}\right) \\
& =\sum_{k \in M_{3}} w^{k}\left(\mu^{\prime}(k)\right)-c\left(\bigcup_{k \in M_{3}} \mu(k) \backslash M_{3}\right)-p\left(\left(\bigcup_{k \in M_{3}} \mu^{\prime}(k) \backslash \bigcup_{k \in M_{3}} \mu(k)\right) \backslash M_{3}\right)+p\left(\left(\bigcup_{k \in M_{3}} \mu(k) \backslash \bigcup_{k \in M_{3}} \mu^{\prime}(k)\right) \backslash M_{3}\right) \\
& =\sum_{k \in M_{3}} w^{k}\left(\mu^{\prime}(k)\right)-c\left(\bigcup_{k \in M_{3}} \mu(k) \backslash M_{3}\right)-c\left(\left(\bigcup_{k \in M_{3}} \mu^{\prime}(k) \backslash \bigcup_{k \in M_{3}} \mu(k)\right) \backslash M_{3}\right)+p\left(\left(\bigcup_{k \in M_{3}} \mu(k) \backslash \bigcup_{k \in M_{3}} \mu^{\prime}(k)\right) \backslash M_{3}\right) \\
& \left.=\sum_{k \in M_{3}} w^{k}\left(\mu^{\prime}(k)\right)-c\left(\bigcup_{k \in M_{3}} \mu^{\prime}(k) \backslash M_{3}\right)-c\left(\left(\bigcup_{k \in M_{3}} \mu(k) \backslash \bigcup_{k \in M_{3}} \mu^{\prime}(k)\right) \backslash M_{3}\right)+p\left(\bigcup_{k \in M_{3}} \mu(k) \backslash \bigcup_{k \in M_{3}} \mu^{\prime}(k)\right) \backslash M_{3}\right) \\
& \geq \sum_{k \in M_{3}} w^{k}\left(\mu^{\prime}(k)\right)-c\left(\mu^{\prime}(k) \backslash\{k\}\right)=\sum_{E \in \mu^{\prime}} v^{w, c}(E),
\end{aligned}
$$

where the first inequality follows from the definition of the demand set and the fact that $(p, \mu)$ is a competitive equilibrium: $w^{k}(\mu(k)) \geq w^{k}\left(\mu^{\prime}(k)\right)-p\left(\mu^{\prime}(k) \backslash\{k\}\right)+p(\mu(k) \backslash\{k\})$. The fourth equality follows from the fact that for all $l \in\left(\bigcup_{k \in M_{3}} \mu^{\prime}(k) \backslash \bigcup_{k \in M_{3}} \mu(k)\right) \backslash M_{3}$, $p_{l}=c_{l}$, and the last inequality follows from the feasibility of the price vector $p$.

Acknowledgements The authors acknowledge the support from research grant ECO201452340-P (Ministerio de Economía y Competitividad), 2014SGR40 and 2014SGR631 (Generalitat de Catalunya).

\section{References}

Davis M, Maschler M (1965) The kernel of a cooperative game. Naval Research Logistics Quarterly 12:223-259

Gale D (1960) The Theory of Linear Economic Models. McGraw-Hill:New York

Hwang YA, Sudhölter P (2001) Axiomatizations of the core on the universal domain and other natural domains. International Journal of Game Theory 29:597-623

Kaneko M, Wooders M (1982) Cores of partitioning games. Mathematical Social Sciences 3:313-327

Keiding H (1986) An axiomatization of the core of a cooperative game. Economics Letters 20:111-115

Llerena F, Núñez M, Rafels C (2015) An axiomatic characterization of the nucleolus of the assignment market. International Journal of Game Theory 44:1-15

Miquel S, Núñez M (2011) The maximum and the addition of assignment games. TOP 19:189-212

Owen G (1992) The assignmen game: the reduced game. Annales d'Economie et de Statistique 25-26:71-79

Peleg B (1986) On the reduced game property and its converse. International Journal of Game Theory 15:187-200

Potters B (1991) An axiomatization of the nucleolus. International Journal of Game Theory 19:365-373 
Quint T (1991a) Necessary and sufficient conditions for balancedness in partitioning games. Mathematical Social Sciences 22:87-91

Quint T (1991b) The core of a $m$-sided assignment game. Games and Economic Behavior 3:487-503

Sasaki H (1995) Consistency and monotonicity in assignment problems. International Journal of Game Theory 24:373-397

Schmeidler D (1969) The nucleolus of a characteristic function game. SIAM Journal of Applied Mathematics 17:1163-1170

Shapley L, Shubik M (1972) The assignment game I: the core. International Journal of Game Theory 1:111-130

Stuart H (1997) The supplier-firm-buyer game and its $m$-sided generalization. Mathematical Social Sciences 34:21-27

Tejada O (2010) A note on competitive prices in multilateral assignment markets. Economics Bulletin 30:658-662

Tejada O (2013) Complements and substitutes in generalized multisided assignment economies. Operations Research Letters 41:468-473

Toda M (2003) Consistency and its converse in assignment games. International Journal of Mathematics, Game Theory and Algebra 13:1-14

Toda M (2005) Axiomatization of the core of assignment games. Games and Economic Behavior 53:248-261 